\title{
Communications
}

\section{Long-reach Optical Access (LROA): A Cost-effective Promising Approach}

\author{
Ibrahim Mohamed \\ Department of Electrical Engineering, Faculty of Engineering, Omar Al-Mukhtar University, Al-Bayda, Libya \\ Email address: \\ engibrahim_2007@yahoo.com

\section{To cite this article:} \\ Ibrahim Mohamed. Long-reach Optical Access (LROA): A Cost-effective Promising Approach. Communications. \\ Vol. 7, No. 2, 2019, pp. 40-44. doi: 10.11648/j.com.20190702.12
}

Received: November 9, 2019; Accepted: November 23, 2019; Published: December 4, 2019

\begin{abstract}
To compensate the decrease in its revenue, most telecom operators have adopted similar strategy which is to provide faster Internet with low cost to its customers. Studies suggested that providing faster Internet with low cost can be achieved by reducing the cost of building next-generation access network. Among the various technologies introduced for next-generation access, long-reach optical access LROA is considered the largest candidate. This is due to the anticipated cost effectiveness of this technology. In LROA, more users can be supported over a common optical component (e.g., a transmitter, a fiber, or probably both), i.e., small number of entities is employed in the access network for service provisioning, which is considered as an improvement in the cost-sharing concept. Our objective in this paper is to verify the cost-effectiveness of this technology. To this end, a statistical-based cost comparison was conducted. The comparison was between the currently deployed passive optical networks (PONs), i.e., the Broad band PON (B-PON [G. 983]), the Ethernet PON (E-PON [IEEE802.3ah]), and the gigabit PON (G-PON [ITU-T G. 984]) and one of the LROA architectures proposed in the literature. The comparison process confirmed that the LROA requires less cost and cost per subscriber as compared with the currently deployed PONs.
\end{abstract}

Keywords: Next Generation Optical Access, Passive Optical Networks PONs, WDM-PONs, Hybrid TDM/WDM-PONs, Long-reach Optical Access

\section{Introduction}

To compensate the decrease in its revenue, most telecom operators have adopted similar strategy which is to provide faster Internet to its customers. However, they will be required to hold out extra cost to do so. Of course customers will pay more for faster Internet, but the amount they are able to pay may not satisfy the operators' aspirations. In other word, the difference between the cost of producing faster Internet and the achieved revenue, which represents the operators' profit, will start to shrink with time. This is called margin erosion [1]. To avoid margin erosion problem, operators are required to either develop new services and applications that attract consumers' attention and thus stimulate them to spend more or find a way to reduce the cost of producing faster Internet. While developing attractive services and applications seems very challenging, reducing the cost of producing faster Internet might be easier and can be achieved if the cost of building the access networks is reduced. In other word, the goal of reducing the cost of producing faster Internet can be realized by simplifying the whole network. Among the various technologies introduced for next-generation access, LROA is a promising solution that ensures simplifying the optical access networks [2]. This is due to the attractive approach adopted in this technology in which a large number of central offices could be consolidated in a single trunk office. Figure 1 clarifies this situation. In LROA, more users can be supported over a common optical component (e.g., a transmitter, a fiber, or probably both), i.e., small number of entities is employed in the network for service provisioning.

This is also can be envisioned as an improvement in the cost-sharing concept. In its early appearance, LROA was basically developed based on Time-Division Multiplexing (TDM) solution in which a single wavelength is employed to serve several Optical Network Units (ONUs). Later, it was introduced as hybrid schemes, i.e., Time-Division Multiplexing/Coarse Wavelength-Division Multiplexing 
[(TDM)/(CWDM)], and Time-Division Multiplexing/Dense Wavelength-Division Multiplexing [(TDM)/(DWDM)]. This paper is devoted to verify the cost-effectiveness of LROA approach that might be achieved by improving the costsharing. To do so, a statistical-based cost comparison was conducted. The comparison was between the currently

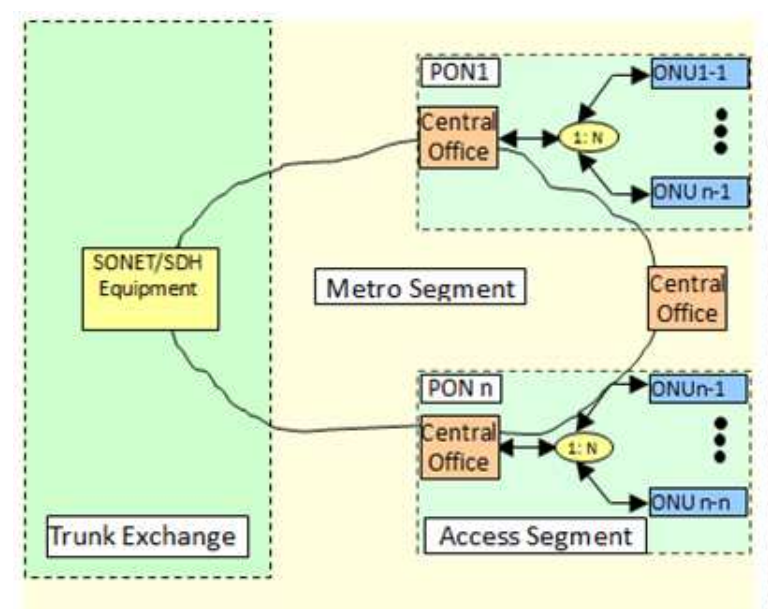

(a) deployed optical access technologies (TDM-based PONs), i.e., the Broad band PON (B-PON [G. 983]), the Ethernet PON (E-PON [IEEE802.3ah]), and the gigabit PON (G-PON [ITU-T G. 984]) and one of the LROA architectures proposed in the literature.

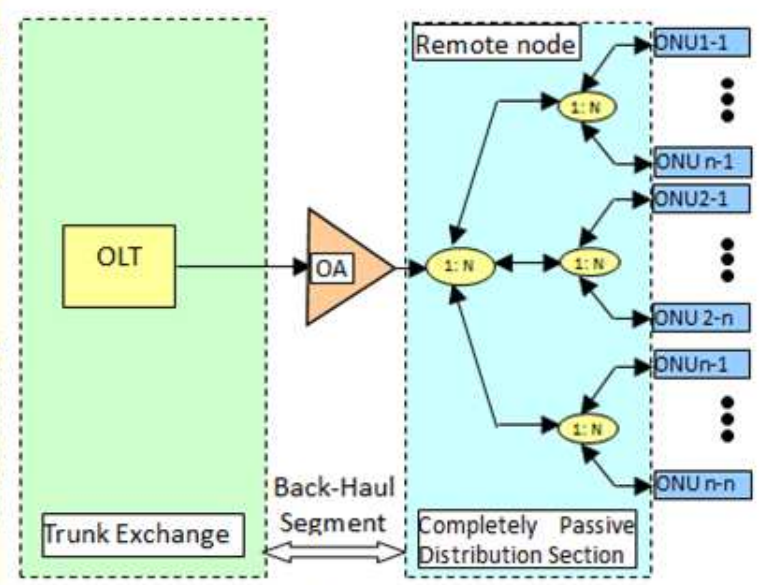

(b)

Figure 1. (a) Basic telecommunication, (b) Simplified LROA architecture.

\section{Next-generation Optical Access}

Higher data rate, larger capacity, and longer range represent specific features a next-generation optical access candidate should at least posses one of them in order to outperform the limitations imposed in the currently deployed optical access technologies [3-8]. A review of enabling technologies for these features are summarized below.

\subsection{High Data Rate TDM-based PONs}

To meet the requirement of higher bit rate that should be fulfilled in next-generation optical access, the Institute of Electrical and Electronics Engineers (IEEE) and the International Telecommunications Union's Telecommunication Standardization Sector (ITU-T) released their standards (IEEE803. av, 10GEPON and ITU-T. 987, XG-PON) in 2009 and 2010, respectively [9, 10]. Both $10 \mathrm{GEPON}$ and XG-PON specify symmetric $10 \mathrm{Gbps}$ for downstream and upstream transmissions. They also specify asymmetric downstream and upstream transmissions. In this aspect, 10GEPON specifies asymmetric transmission with 10 Gbps for downstream and $1 \mathrm{Gbps}$ for upstream, respectively; whereas, XG-PON specifies asymmetric transmission with 10 Gbps for downstream and $2.5 \mathrm{Gbps}$ for upstream, respectively.

\subsection{Wavelength Division Multiplexing WDM-based PONs}

Wavelength Division Multiplexing WDM-based PONs were basically introduced to exploit the large number of wavelength an optical fiber can carry (its virtual unlimited bandwidth), which leads to increase the system capacity, i.e., were proposed to meet the requirement of larger capacity that should be fulfilled in next-generation optical access. Examples of WDM-based PONs can be found in [11- 14]. Compared with TDM, WDM provides more privacy and security as each ONU in the PON system can use a single and dedicated pair of wavelength. Another worth-mentioning feature is that WDM leads to facilitate coexistence among different operators; i.e., new operators can share the same Optical Distribution Network (ODN) with legacy ones. As addressing the advantages and disadvantages of WDM is beyond the scope of this paper, detailed information on that can be found in $[15,16]$.

\subsection{Hybrid TDM/WDM-based PONS}

Hybrid TDM/WDM-based PONs were basically introduced as a solution to integrate and exploit the features offered by both TDM-based PONs and WDM-based PONs. On other word, a high data rate and huge capacity PON system can be developed in one hybrid scheme. Based on the specified wavelength spacing, hybrid TDM/WDM-based PONs can be categorized as either TDM/CWDM-based PONs or TDM/DWDM-based PONs. While hybrid TDM/CWDM-based PONs specify $20 \mathrm{~nm}$ for wavelength spacing, hybrid TDM/DWDM-based PONs specify either 0.8 or $0.4 \mathrm{~nm}$ for wavelength spacing. Hybrid TDM/WDM-based PONs can also be categorized as either static or dynamic schemes. In static schemes, a dedicated pair of wavelengths is allocated to each ONU for upstream and downstream transmissions; whereas in dynamic schemes, wavelengths are allocated dynamically during communication, i.e., each pair of wavelengths can be allocated several times and serve multiple ONUs. Examples of hybrid TDM/CWDM-based PONs and hybrid TDM/DWDM-based PONs can be found in [14-21]. 


\subsection{Long-Reach Optical Access (LROA)}

LROA was basically introduced as a solution to overcome certain limitations imposed in the currently deployed PONs, i.e., the small capacity and short range. It offers an attractive solution by which more users can be supported over a common infrastructure, which improves the cost-sharing and the efficiency of the access system. To extend the range and increase the capacity, solutions based on signal amplification would be required. Hence, the need for using amplifiers in LROA becomes substantial and inevitable. In the LROA, optical amplifiers are suggested to be employed in the field instead of conventional repeaters. The advantage of this approach is that optical amplifiers work completely in the optical domain, which leads to omit involving complex and expensive processes, such as photon-to-electron conversion, retiming, reshaping, electrical amplification, and electron tophoton conversion. A further advantage is that optical amplifiers are transparent to the bit-rate changes and the data format used. In its early appearance, LROA schemes were basically developed based on TDM solution in which a single wavelength is employed to serve several ONUs. Later, they were proposed as hybrid TDM/CWDM, or TDM/DWDM schemes. Examples of several LROA schemes can be found in [22-32].

\section{Statistical-based Cost Comparision}

To verify the cost-effectiveness of LROA, a statisticalbased cost comparison was conducted. The comparison was between the currently deployed optical access technologies (TDM-based PONs), i.e., the Broad band PON (B-PON [G. 983]), the Ethernet PON (E-PON [IEEE802.3ah]), and the gigabit PON (G-PON [ITU-T G. 984]) and one of the LROA architectures proposed in the literature. The selected LROA architecture was proposed by the author in [30]. In this proposed architecture, the capacity was increased as a result of increasing the splitting ratio, which helps improve the cost sharing. The proposed LROA architecture contains five zones; each zone is capable to support 768 users. Tow different approaches were followed for service provisioning (single-fiber or multi-fiber approach). In single-fiber approach, a single fiber was employed for service provisioning to each zone, whereas in multi-fiber approach, three fibers were employed for service provisioning to each zone. The optical equipments required to support each zone and its corresponding cost are listed bellow. The cost of the optical components was obtained through Internet by accessing Fiberstore website (www.fiberstore.com).

In Case of Adopting Single-fiber approach:

a) Three DWDM transceivers, Cost $=3 \times 286$ USD $=858$ USD

b) One optical fiber cables that contains 2 SMFs $($ cost $/ \mathrm{meter}=0.42 \mathrm{US} / \mathrm{m})$, Cost $=1 \times(0.37 \mathrm{US} / \mathrm{m} \times 20 \times$ $103 \mathrm{~m})=7,400$ USD

c) Three $(1 \times 4)$ power splitters, Cost $=3 \times 11.70 \mathrm{USD}=$ 35.1 USD d) Three $(1 \times 64)$ power splitters, Cost $=3 \times 59$ USD $=$ 177 USD

e) One EDFA, Cost $=1 \times 1842$ USD $\rightarrow$ EDFA cost $/$ zone $=$ $1 \times 1842 / 5=368.4$ USD

f) 16 Channels AWG DWDM Mux/Demux, Cost $=1 \times$ 1104 USD $\rightarrow$ Cost $/$ zone $=1104$ USD $/ 5=220.8$ USD

g) Two of 4 channel AWG DWDM Mux/Demux, Cost $=2$ $\times 276$ USD $=552$ USD

The total cost needed to support each zone $=9,611.3$ USD, which yielding Cost/user $=12.5$ USD

In Case of Adopting Multi-fiber approach:

a) Three DWDM transceivers, Cost $=3 \times 286$ USD $=858$ USD

b) One optical fiber cables that contains 4 SMFs $($ cost $/$ meter $=0.42 \mathrm{US} / \mathrm{m})$, Cost $=1 \times(0.42 \mathrm{US} / \mathrm{m} \times 20$ $\times 103 \mathrm{~m})=8,400 \mathrm{USD}$

c) Three $(1 \times 4)$ power splitters, Cost $=3 \times 11.70 \mathrm{USD}=$ 35.1 USD

d) Three $(1 \times 64)$ power splitters, Cost $=3 \times 59$ USD $=$ 177 USD

e) One EDFA, Cost $=1 \times 1842$ USD $\rightarrow$ EDFA cost $/$ zone $=$ $1 \times 1842 / 5=368.4$ USD

f) 16 Channels AWG DWDM Mux/Demux, Cost $=1 \times$ 1104 USD $\rightarrow$ Cost $/$ zone $=1104$ USD $/ 5=220.8$ USD

The total cost needed to support each zone $=10,059.3$ USD, which yielding Cost/user $=13.09$ USD. The optical equipments and its corresponding cost required to support an equivalent number of users that can be supported in each zone in the selected LROA architecture in case of using currently deployed PONs are listed bellow.

In case of using B-PON, 16 user/pon segment:

a) Forty eight transceivers, Cost $=48 \times 65$ USD $=3,120$ USD

b) Four optical cables with $12 \mathrm{SMFs}$ for each $($ cost $/$ meter $=$ $0.61 \mathrm{USD} / \mathrm{m})$, Cost $=4 \times(0.61 \mathrm{USD} / \mathrm{m} \times 20 \times 103 \mathrm{~m})=$ 48,800 USD

c) Forty eight $(1 \times 16)$ power splitters, Cost $=48 \times 16.2$ $\mathrm{USD}=766.6 \mathrm{USD}$

The total cost needed $=52,686.6$ USD, which yielding Cost $/$ user $=68.6$ USD.

In case of using E-PON, 32 user/pon segment:

a) Twenty four transceivers, Cost $=24 \times 65$ USD $=1560$ USD

b) Two optical cables with $12 \mathrm{SMFs}$ for each (cost $/$ meter = $0.61 \mathrm{USD} / \mathrm{m})$, Cost $=2 \times(0.61 \mathrm{USD} / \mathrm{m} \times 20 \times 103 \mathrm{~m})=$ 24,400 USD

c) Twenty four $(1 \times 32)$ power splitters, Cost $=24 \times 21.67$ USD $=520.08$ USD

The total cost needed $=26,480.08$ USD, which yielding Cost $/$ user $=34.47$ USD.

In case of using G-PON, 64 user/pon segment:

a) Twelve transceivers, Cost $=12 \times 79$ USD $=948$ USD

b) One optical fiber cable with $12 \mathrm{SMFs}$ for each $(\mathrm{Cost} / \mathrm{m}$ $=0.61 \mathrm{USD} / \mathrm{m}), \mathrm{Cost}=1 \times(0.61 \mathrm{USD} / \mathrm{m} \times 20 \times 103 \mathrm{~m})$ $=12,200 \mathrm{USD}$

c) Twelve $(1 \times 64)$ power splitters, $12 \times 59$ USD $=708$ USD 
The total cost needed $=13,856$ USD, which yielding Cost/user $=18.04$ USD. To simplify the comparison process, the collected data were converted to charts using Excel. The total cost required in each access scheme to support 768 users, and the cost per user is shown in Figure 2.
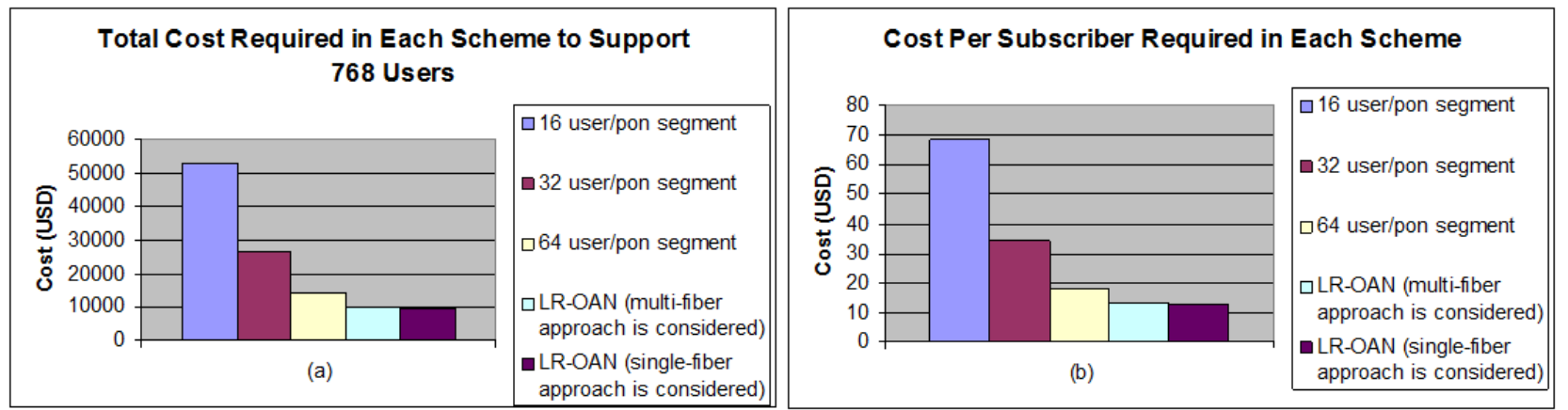

Figure 2. (a) Total cost required in each scheme to support 768 users, (b) Cost per subscriber required in each scheme.
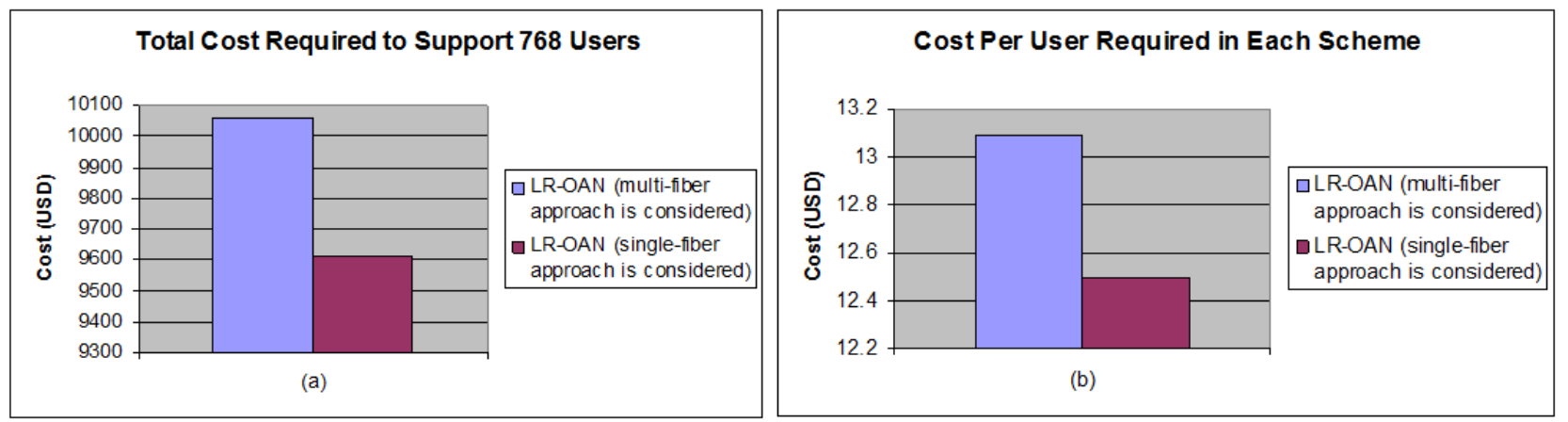

Figure 3. (a) Total cost required to support 768 users when single-fiber LROA and multi-fiber LROA are used, (b) Cost per subscriber required in each scheme.

It can be obviously seen from the charts that the long-reach access requires less cost as well as requires less cost per subscriber as compared with the currently deployed PONs. The charts also show that the single-fiber LROA approach requires the lowest cost and cost per subscriber. Figure 3 was included to show this observation clearly.

\section{Conclusions}

The cost-effectiveness of LROA was verified using statistical-based comparison. The optical equipments required to support a number of 768 users and its corresponding cost in case of using LROA and each of the PON systems deployed currently (B-PON, E-PON, and G-PON) were involved in the comparison. The comparison process confirmed the costeffectiveness of the LROA as it requires less cost per subscriber compared with the currently deployed PONs. Specifically, the cost per subscriber was 12.5 USD in case of using LROA which was the lowest compared with that required in case of using B-PON, E-PON, or G-PON, which was 68.6 USD, 34.47 USD, or 18.04 USD, respectively.

\section{References}

[1] Josep Prat. Next-Generation FTTH Passive Optical Networks. Springer Science, Business Media B. V. 2008.
[2] Ibrahim Mohamed and Mohammad Syuhaimi, LONGREACH OPTICAL ACCESS NETWORKS (LR-OANS): A PROMISING CANDIDATE FOR FUTURE OPTICAL ACCESS, American Journal of Applied Sciences 11 (9): 16041615, 2014.

[3] Ibrahim Mohamed and Mohammad Syuhaimi, Options and challenges in next-generation optical access networks (NGOANs), Optik 126 (2015) 131-138.

[4] Jianhe Gao, "Demonstration of the first $29 \mathrm{~dB}$ power budget of $25-\mathrm{Gb} / \mathrm{s}$ 4-PAM system without optical amplifier for next generation access network", Optical Fiber Communications Conference and Exhibition (OFC), 2016.

[5] Richard M. Dorward, Michael J. Anderson, and Roger P. Giddings, "Technical and market feasibility of high-speed software-reconfigurable OOFDM/DFMA-based Optical transceivers for Next Generation Access Network PONs", 18th International Conference on Transparent Optical Networks (ICTON), 2016.

[6] Zhenhua Feng, Ming Tang, Xun Guan, Calvin Chun-Kit Chan, Qiong Wu, Xi Chen, Ruoxu Wang, Rui Lin, Songnian Fu, Lei Deng, and Deming Liu "Spectrally overlaid DDO-OFDM transmission enabled by optical power division multiplexing", 15th International Conference on Optical Communications and Networks (ICOCN), 2016.

[7] Zhenhua Feng; Liang Xu; Qiong Wu; Ming Tang; Songnian Fu; Weijun Tong; Deming Liu "Large-capacity optical access network utilizing multicore fiber and self-homodyne coherent detection", Optical Fiber Communications Conference and Exhibition (OFC), 2017. 
[8] Xinhua Zhu, Mengfan Cheng, Lei Deng, XingXing Jiang, Changjian Ke, Minming Zhang, Songnian Fu, Ming Tang, Ping Shum, and Deming Liu "An Optically Coupled ElectroOptic Chaos System With Suppressed Time-Delay Signature", IEEE Photonics Journal, Volume: 9, Issue 3, 2017.

[9] IEEE Std 802.3av-2009, (Amendment to IEEE Std 802.32008), "Physicallayer specifications and management parameters for $10 \mathrm{~Gb} / \mathrm{s}$ passive opticalnetworks," Approved 11 September 2009, IEEE-SA Standards Board.

[10] ITU-T Recommendation G. 987 series: Digital sections and digital line sys-tem - Optical line systems for local and access networks, "10-Gigabit-capablepassive optical network (XGPON) systems: Definitions, abbreviations andacronyms", 2010.

[11] H. Takesue, T. Sugie, Wavelength channel data rewrite using saturatedSOAmodulator for WDM networks with centralized light sources, IEEE J. Light-wave Technol. 21 (Nov (11)) (2003) 2546-2556.

[12] N. Genay, P. Chanclou, T. Duong, N. Brochier, and E. Pincemin, "BidirectionalWDM/TDM- PON access networks integrating downstream $10 \mathrm{Gbit} / \mathrm{s}$ DPSK andupstream 2.5 Gbit/s OOK on the same wavelength", Proceeding of The EuropeanConference on Optical Communications (ECOC 2006), Cannes, France, 24-28Sept 2006, pp. 1-2.

[13] H. Suzuki, M. Fujiwara, T. Suzuki, N. Yoshimoto, H. Kimura, M. Tsubokawa, Wavelength-tunable DWDM-SFP transceiver with a signal monitoring inter-face and its application to coexistence-type colorless WDM-PON, in: Presentedat the 33rd Eur. Conf. Exhib. Opt. Commun. (ECOC 2007), Sept. 16-20, Berlin, Germany, 2007.

[14] J. Kani, K. Iwatsuki, A wavelength-tunable optical transmitter using semicon-ductor optical amplifiers and an optical tunable filter for metro/access DWDMapplications, IEEE J. Lightwave Technol. 23 (Mar (3)) (2005) 1164-1169.

[15] K. Grobe, J. P. Elbers, PON in adolescence: from TDMA to WDM-PON, IEEE Com-mun. Mag. 46 (2008) 26-34.

[16] J. i. Kani, Enabling technologies for future scalable and flexible WDM-PONand WDM/TDM-PON systems, IEEE J. Sel. Top. Quantum Electron. 16 (2010) 1290-1297.

[17] Jie Hyun Lee, Seung-Hyun Cho, Han-Hyub Lee, Eui-Suk Jung, Jea-Hoon Yu, Byoung-Whi Kim, Sang-Hyeun Lee, JaiSang Koh, Back-Heung Sung, Suk-JinKang, Jin-Hee Kim, KiTae Jeong, Sang Soo Lee, First commercial deploymentof a colorless gigabit WDM/TDM hybrid PON system using remote protocolterminator, J. Lightwave Technol. 28 (2010) 344-351.

[18] Yuanqiu Luo, Xiaoping Zhou, Frank Effenberger, Xuejin Yan, Guikai Peng, Yinbo Qian, Yiran Ma, Time- and wavelengthdivision multiplexed passive opticalnetwork (TWDM-PON) for next-generation PON stage 2 (NG-PON2), J. Light-wave Technol. 31 (February (4)) (2013) 587-593.

[19] Abhishek Dixit, Bart Lannoo, Goutam Das, Didier Colle, Mario Pickavet, PietDemeester, Flexible TDMA/WDMA passive optical network: Energy efficientnext-generation optical access solution, Opt. Switching Networking 10 (4) (2013) 491-506.
[20] Mohammad Syuhaimi, Ibrahim Mohamed, High efficient fiber plant utiliza-tion by multiple PON infrastructure based on frequency re-use approach forscalable FTTH networks, in: Presented at the Third Global Conference for Aca-demic Research on Scientific and Emerging Technologies GCARSET, March. 9-11, Kuala Lumpur, Malaysia, 2013.

[21] A. Kaneko, T. Goh, H. Yamada, T. Tanaka, L. Ogawa, Design and applicationsof silica-based planar lightwave circuits, IEEE J. Sel. Top. Quantum Electron. 5 (Sept/Oct (5)) (1999) 1227 1236.

[22] I. Van de Voorde, C. M. Martin, J. Vandewege, X. Z. Qiu, The SuperPON demon-strator: an exploration of possible evolution paths for optical access networks, IEEE Commun. Mag. 38 (2000) 74-82.

[23] D. P. Shea, J. E. Mitchell, A 10 Gb/s 1024-way split 100-km long reachoptical access network, J. Lightwave Technol. 25 (Mar (3)) (2007) 685-693.

[24] P. P. Iannone, H. H. Lee, K. C. Reichmann, X. Zhou, M. Du, B. Pálsdóttir, K. Feder, P. Westbrook, K. Brar, J. Mann, L. Spiekman, Four extended-reach TDM PONs shar-ing a bidirectional hybrid CWDM mplifier, J. Lightwave Technol. 26 (January (1)) (2008) 138-143.

[25] P. P. Iannone, K. C. Reichmann, X. Zhou, S. Gray, A discrete SOA-Raman hybridamplifier with $80-\mathrm{nm}$ bandwidth, in: Proc. ECON, 2006, pp. 1-2.

[26] H. Lee, P. Iannone, K. Reichmann, J. Lee, B. Palsdottir, A C/L-band gain-clampedSOA-Raman hybrid amplifier for CWDM access networks, IEEE Photonics Tech-nol. Lett. 20 (3) (2008) 196-198.

[27] G. Talli, P. D. Townsend, Hybrid DWDM-TDM long-reach PON for next-generation optical access, J. Lightwave Technol. 24 (2006) 2827-2834.

[28] D. P. Shea, J. E. Mitchell, Experimental upstream demonstration of a long reach wavelength-converting PON with DWDM Backhaul, in: Proc. Optical Fiber Communication and the National Fiber Optic Engineers Conference, OFC/NFOEC, 2007, pp. 1-3.

[29] Minwan Jung, You Min Chang, Ju Han Lee, A band-separated, bidirec-tional amplifier based on erbium-doped bismuth fiber for long-reach hybrid DWDM-TDM passive optical networks, J. Opt. Commun. Networking 4 (3) (2012).

[30] Mohammad Syuhaimi, Ibrahim Mohamed, Highly utilized fiber plant with extended reach and high splitting ratio based on AWG and EDFA characteristics, ETRI J. 35 (5) (2013) 786-796.

[31] Huan Song, Byoung-Whi Kim, Biswanath Mukherjee, Multithread polling: adynamic bandwidth distribution scheme in long-reach PON, IEEE J. Sel. Areas Commun. 27 (February (2)) (2009) 134-142.

[32] L. G. Kazovsky, S.-W. Wong, V. Gudla, P. T. Afshar, S.-H. Yen, S. Yamashita, Y. Yan, Challenges in next-generation optical access networks: addressing reach extension and security weaknesses, IET Optoelectron. 5 (4) (2011) 133-143. 